\title{
Un acercamiento a las minorías universitarias
}

\section{An approach to university minorities}

\author{
Higuera Bonfil, Antonio (coord.) (20 io), Creer y APRENDER. \\ RETRATOS CULTURALES DE ESTUDIANTES UNIVERSITARIOS, UNIVERSIDAD \\ de Quintana Roo-Plaza y Valdés, México, 365 Pp., \\ ISBN: 978-607-402-203-2.
}

La entrada a un nuevo espacio educativo siempre genera incertidumbre: requiere de un proceso de adaptación social, implica conocer nuevos compañeros, enfrentarse a nuevos procesos de aprendizaje, a obligaciones distintas. Sin embargo la adaptación no es igual para todos los involucrados, depende de la experiencia del alumno, del sexo, de la edad, de los motivos para estudiar, pero también -como se muestra en este libro- del lugar de procedencia, de la condición étnica y de la religión que profesan.

El subtítulo del libro, Retratos culturales de estudiantes universitarios, nos remite precisamente a su contenido. Se trata de tres estudios y nueve historias de vida hechas a jóvenes estudiantes -tres hombres y seis mujeres- de grupos minoritarios. Son tres universos distintos que dibujan un retrato cultural que vincula al individuo, a cada joven, con lo colectivo.

El primer estudio relata el caso de tres jóvenes taiwaneses que estudiaron la educación básica en su país y llegan a Belice con el objetivo de estudiar inglés, posteriormente viajan a México para inscribirse en los estudios superiores en lenguas inglesas en la Universidad de Quintana Roo; el segundo texto nos abre una puerta a la vida actual de los mayas a través de tres jóvenes - dos mujeres y un hombre- quienes, con una vitalidad desbordante, cuentan su paso por la primaria, la secundaria y el bachillerato hasta que logran ser aceptados en la Universidad de Quintana Roo; finalmente, el último análisis nos lleva a un mundo para muchos poco conocido, el de los testigos de Jehová, a través de los ojos y el habla de tres chicos, los tres convencidos del peso prioritario que la religión tiene en sus vidas.

A continuación se exponen, en un primer apartado, grosso modo, los hallazgos que los tres investigadores, María del Rosario Reyes Cruz, Griselda Murrieta Loyo y Antonio Higuera Bonfil -académicos de la Universidad de Quintana Roo, unidad Chetumal-consideran más relevantes y en un segundo bloque hago una breve reflexión a partir de temas transversales que fueron resultado de la lectura del libro. 


\section{Hallazgos del libro}

María del Rosario Reyes Cruz analiza tres historias de vida de jóvenes taiwaneses que estudian en la Universidad de Quintana Roo, en Chetumal, su objetivo es investigar las creencias epistemológicas y lingüísticas de los estudiantes taiwaneses y así entender la manera en que ellos conciben el aprendizaje y la enseñanza, en especial en la carrera que cursan: letras inglesas. Las tres historias tienen rasgos comunes: los tres salen de Taiwán rumbo a Belice para aprender inglés, pues es un idioma fundamental para la vida laboral en su país. Sin embargo, después de cruzar el mundo se dan cuenta que no aprenderán mucho inglés pues los beliceños se comunican más bien en criollo. Unos viajan a Belice solos (como Kuang, el hombre entrevistado), las mujeres migran acompañadas (una con una hermana y otra con toda su familia), posteriormente se dirigen a Chetumal para realizar estudios universitarios. Para los tres el proceso de aprendizaje en México resulta complicado, pues deben entenderlo inmersos en una cultura completamente distinta.

La autora encuentra que las dificultades que presentan estos jóvenes tienen que ver no sólo con su limitado conocimiento del español, sino con las creencias propias del mundo oriental en las que fueron formados en su infancia, como asumir que el maestro es la fuente del conocimiento, por tanto no se le cuestiona y jamás se le critica. Pero sus problemas escolares se potencian ante las expectativas que sus familias pusieron en ellos. Fueron enviados al extranjero a estudiar debido a la competencia en el terreno laboral en su país, que los orilla a avanzar en el terreno educativo para no quedarse completamente rezagados en el laboral, para ellos lo importante es aprobar y obtener una buena calificación, el objetivo de su viaje a Chetumal fue obtener un título, eso es lo importante y no necesariamente el aprender.

Los jóvenes taiwaneses lo que conocen para aprender es memorizar y no analizar y eso, en el proceso de aprendizaje del ambiente educativo mexicano les genera inconvenientes, pues debido a que las estrategias didácticas de las universidades mexicanas son más flexibles y requieren de participación activa, ellos se sienten y están en desventaja.

A estos taiwaneses los liga el haber migrado de un país culturalmente muy diferente y el tener que comunicarse en lengua española, pero también los une el empeño, el esfuerzo triple que realizan cada día (con respecto al idioma, al proceso de aprendizaje y con la convivencia diaria en una cultura completamente distinta) y la necesidad imperiosa de no fracasar.

Graciela Murrieta escribe, en el segundo apartado del libro, acerca de los estudiantes mayas. La autora investiga el significado que tiene para los jóvenes el conocimiento y el aprendizaje, y señala a su vez cómo ellos 
perciben el papel de los maestros y alumnos de la comunidad de la Universidad de Quintana Roo. Murrieta presenta la historia de vida de dos mujeres y de un hombre. Se trata de jóvenes procedentes de pequeñas comunidades cercanas a Chetumal, donde hicieron sus estudios previos a la universidad, el que provengan de poblados pequeños y aislados los marcó y limitó, pues consideran que su formación básica ha sido elemental. ${ }^{1}$ Los tres comparten el conocimiento del maya (dos lo hablan y entienden, una sólo lo entiende), ${ }^{2}$ pero más que eso, comparten una tradición y una cosmovisión producto de su origen étnico. Murrieta, citando a Chin y Brewer, señala que no está del todo claro que la educación cause un cambio en el tipo de creencias que una persona posee. Ella supone que la educación puede transformar activamente las creencias a partir de proveer a los estudiantes más información, motivarlos a cuestionar y provocar el análisis de éstas en el momento en que se enfrentan a los problemas. En este caso los tres jóvenes asumen su origen étnico con gran orgullo y su filosofía de vida maya parece haber permeado las creencias epistemológicas y del aprendizaje. Murrieta concluye que sus creencias, lejos de ser un obstáculo en el proceso de aprendizaje, han sido un factor fundamental en el desarrollo de buenas estrategias en el momento de aprender.

A lo largo de las entrevistas, los tres jóvenes mayas dejan ver su inteligencia y su deseo de aprender cada vez más. Para ellos la disciplina, la responsabilidad y el esfuerzo son la base para avanzar en sus estudios, pero además han comprendido que el conocimiento es un proceso escalonado, hay que ir aprendiendo paso a paso y desde el principio.

Contrario a sus compañeros de Taiwán, para ellos el maestro es importante pero les queda claro que existen otros recursos complementarios, como los libros y el apoyo de los pares. Pero sobre todo han asumido que son capaces de hacer las cosas bien y de crearse nuevas metas.

El análisis que concluye el libro corresponde a Antonio Higuera Bonfil, quien también es el coordinador de la investigación que dio origen a esta publicación. En este apartado el autor incluye tres historias de vida (también de dos mujeres y un hombre) que pertenecen a una minoría religiosa, los testigos de Jehová, ${ }^{3}$ y busca conocer si esta adscripción religiosa es una variable que influye en el desempeño académico de los jóvenes. Higuera señala que el sistema de creencias de los testigos de Jehová los lleva a un compromiso que va más allá de la asistencia a los servicios

${ }^{1}$ Según el Conteo de Población 2005, sólo 27 de cada 100 quintanarroenses concluyeron la educación secundaria y sólo 15 de cada 100 terminaron la educación media superior y pudieron optar por continuar estudios universitarios.

${ }^{2}$ Las cifras del Conteo 2005 señala que en Quintana Roo 19\% de sus habitantes habla alguna lengua indígena.

${ }^{3}$ En el Censo de 2000 se informa que en Quintana Roo 2.23\% de la población es testigo de Jehová (Higuera, 2006). 
religiosos, de ahí la intención de explorar los espacios y tiempos que ellos dan a las diversas esferas de su quehacer cotidiano: la universidad y sus tareas, la vida social, la vida laboral, el descanso y las actividades de orden religioso.

Los tres entrevistados son miembros activos de su congregación, por lo que predican regularmente, dirigen estudios bíblicos y uno, el hombre entrevistado, realiza muchas más actividades relacionadas con su religión.

A partir del uso de genealogías como herramienta de análisis, el autor muestra que la pertenencia a este grupo religioso, sobre todo en el caso de las dos jóvenes, es una forma de vida familiar que abarca varias generaciones, no se trata de dos muchachas que practican una religión distinta a la católica, ${ }^{4}$ sino de dos mujeres jóvenes cuyos elementos ideológicos religiosos les han sido dados desde el entorno familiar y desde siempre.

Los hallazgos muestran que, en efecto, la vida de los tres entrevistados se rige por los lineamientos establecidos por la Sociedad Torre del Vigía. ${ }^{5}$ $\mathrm{Su}$ actividad primordial tiene que ver con los servicios religiosos, los cuales organizan de manera puntual y rigurosa; y no sólo eso, sino que su presencia en la educación superior, si bien tiene que ver con la posibilidad de obtener un buen empleo al concluir su carrera, la intención de un buen trabajo está vinculada con ampliar las posibilidades para desempeñar, en mejores condiciones, sus diferentes y cada vez más amplias responsabilidades religiosas. ${ }^{6}$

En relación con el proceso de aprendizaje, se trata de alumnos estudiosos, responsables y muy trabajadores, pues ser un testigo de Jehová los obliga a asumir con seriedad lo que se proponen. Para ellos su desempeño académico está fundamentado en el hecho de saber que un testigo de Jehová debe rendir testimonio de su fe en todas sus actividades cotidianas. Sin embargo, se sienten excluidos o más bien se excluyen del ambiente universitario porque en ocasiones lo consideran vulgar, pecaminoso, poco sano, incorrecto. Esta condición de excluido que a cualquier joven universitario le haría sentirse profundamente deprimido -en la medida en que la importancia de la socialidad entre los jóvenes es precisamente el disfrute de estar juntos y convivir de manera horizontal, es decir, sin jerarquías (Mafessoli, 2002)-, a ellos no les afecta, de hecho lo asumen como un proceso normal, se saben y se sienten distintos. No es que estén aislados en general, sólo que los espacios de estos tres jóvenes para socializar no los obtienen vía la universidad, sino que la identificación con los otros -importantísima en la etapa juvenil- la encuentran en su verdad religiosa.

\footnotetext{
${ }^{4}$ En el año 2000, 73.16\% de los quintanarroenses eran católicos (Higuera, 2006).

${ }^{5}$ Organización central de los testigos del Jehová.

${ }^{6}$ Las cifras del Censo del año 2000 muestran que sólo 2 de cada 100 testigos de Jehová ha cursado estudios superiores.
} 


\section{Reflexiones sobre el libro}

El libro Creer y aprender nos remite a grupos de población poco estudiados, a minorías: taiwaneses en el sureste de México, mayas en Quintana Roo, testigos de Jehová en Chetumal, pero más aún, a minorías en una institución cuyo acceso también es limitado: según datos en la introducción del libro, la Universidad de Quintana Roo en el ciclo 2007-2008 tenía 3,260 alumnos; según cifras del Censo de 2000, sólo 8 de cada 100 quintanarroenses finalizaron la educación profesional y $1 \%$ tenía estudios de posgrado.

En el libro se enmarca en un aparente todo qué es la universidad para tres construcciones identitarias heterogéneas: universitarios taiwaneses, mayas y testigos de Jehová; se analiza a jóvenes que confluyen en un mismo lugar pero con distintos estilos, diferentes procesos y diversas trayectorias.

Esto permite corroborar que la juventud no es un sector inerte, que es polisémico y cambiante y que su comprensión implica conocer sus adscripciones en este mundo complejo y crecientemente globalizado (Valenzuela, 2009: 34-35).

En cierto sentido, desde mi punto de vista, para algunos de los jóvenes que participaron en este estudio su paso por la universidad es lo menos importante, de hecho, ser universitarios es sólo una identidad transitoria (para los taiwaneses y los testigos de Jehová); en cambio, los jóvenes mayas se han apropiado de la vida universitaria y piensan, hacen y aplican el conocimiento a pesar de las deficiencias educativas, sociales y económicas que los han limitado. El significado que los tres grupos de jóvenes entrevistados tienen de la educación superior es distinta, lo que se comparte es la pertenencia a un espacio donde estudian. De ahí que además de mostrar las diferencias en el proceso de aprendizaje de estos tres grupos, un punto relevante del estudio ha sido explicar cómo a partir de la presencia en la universidad, las articulaciones y los contextos sociales mueven con sentidos a veces opuestos la vida de estos estudiantes.

Las historias contadas muestran que para unos el título universitario es un mero trámite para no defraudar a los padres (una taiwanesa); para otros es lo que marcará la entrada a la vida laboral en condiciones menos adversas, pero abrirá además un nuevo mundo o al menos una manera distinta de mirar al mundo (los mayas); y para otros es el paso necesario para apoyar en mejores condiciones a su congregación religiosa o bien es un acto que hay que cumplir como un deber (testigos de Jehová).

Otro elemento que llama la atención a lo largo de la lectura es el tema del esfuerzo personal de los jóvenes y sus familias. Hoy día los periódicos, y las noticias en general, a lo que remiten cuando se habla de este grupo etario es a jóvenes en condiciones de riesgo, de vulnerabilidad y de exclu- 
sión (violencia, drogadicción, desempleo); si bien es cierto que muchos han tenido sólo esas opciones, en las historias que cada uno de los jóvenes contó y que forman este libro, no surgen solamente las desventajas acumulativas que ahora están conformando la vida de muchos jóvenes. Aparece un elemento clave que es la condición familiar de la que parten. Todos, excepto uno, tienen en su unidad doméstica, pero fundamentalmente en las madres de familia, el apoyo solidario y material para continuar estudiando, para la construcción de su proyecto de vida.

En Creer y aprender. Retratos culturales de estudiantes universitarios se nos muestra que en contextos socioculturales complejos que enmarcan los grandes cambios de las sociedades contemporáneas, todavía hay jóvenes donde el sentido atribuido a la escuela es positivo y forma parte del proyecto de vida, se evidencia que hay un grupo de jóvenes que conservan cualidades semejantes, como el deseo de aprender, y permanentemente están en la búsqueda de herramientas personales para lograr el aprendizaje en la escuela y también en la vida.

\section{Bibliografía}

Censo General de Población y Vivenda (2000), <http://www.inegi.org. $\mathrm{mx} /$ sistemas/olap/Proyectos/bd/censos/cpv2000/P5.asp?s=est\&c= 10262\&proy=cpv00_p5>, 26 de octubre de 2010.

Conteo de Población y Vivienda (2005), <http://cuentame.inegi.gob.mx/ monografias/informacion/qroo/poblacion/educacion.aspx?tema= me\&e=23>, 26 de octubre de 2010.

Higuera-Bonfil, Antonio (2006), "Religión y construcción de la familia. El caso de los testigos de Jehová", en Antonio Higuera Bonfil (coord.) Trabajo de campo. La antropología en acción, Plaza y ValdésUniversidad de Quintana Roo, México, pp. 91-110.

Maffesoli, Michel (2002) "Tribalismo posmoderno. De la identidad a las identificaciones", en Aquiles Chihu (coord.), Sociología de la identidad, Porrúa, México, pp. 223-242.

Valenzuela, José Manuel (2009), “Introducción. Decálogo para repensar las certezas", en El futuro ya fue, El Colegio de la Frontera NorteCasa Juan Pablos, México, pp. 19-54. 
Recibida: 29 de octubre de 2010. Aceptada: 30 de noviembre de 2010.

Emma LiLiana Navarrete El Colegio Mexiquense Correo-e: enavarr@prodigy.mx

Emma Liliana Navarrete. Es antropóloga social por la Universidad Autónoma Metropolitana, Iztapalapa; maestra en demografía por El Colegio de México y doctora en estudios de población también por El Colegio de México. Actualmente es profesora-investigadora en El Colegio Mexiquense. Sus principales áreas de investigación son los jóvenes, el trabajo y el entorno laboral de mujeres universitarias. Sus publicaciones más recientes son: "Nivel de escolaridad y oportunidades laborales para las mujeres mexiquenses al inicio del siglo xxI", en Emma Liliana Navarrete (coord.), Mujeres mexiquenses. Pasado y presente de las voluntades que transforman, Biblioteca Mexiquense del Bicentenario, Toluca, pp. 237-261 (2009); "Trabajo y universitarias. Mujeres mexiquense de dos generaciones", en Luz María Salazar (coord.), Sujetos laborales del Estado de México, El Colegio Mexiquense, Zinacantepec, pp. 153-175 (2009). 